\title{
Effect of Tirapazamine and Mild Temperature Hyperthermia on the Recovery from Radiation-Induced Damage in Pimonidazole-Unlabeled Quiescent Tumor Cell Population
}

\author{
Shin-Ichiro Masunaga*, Yoshinori Sakurai, Hiroki Tanaka, Minoru Suzuki, Natsuko Kondo, \\ Masaru Narabayashi, Keizo Tano, Akira Maruhashi, Koji Ono \\ Radiation Life and Medical Science Research Division, Research Reactor Institute, Kyoto University, Kyoto, Japan. \\ Email: *smasuna@rri.kyoto-u.ac.jp \\ Received February $14^{\text {th }}, 2013$; revised March $16^{\text {th }}, 2013$; accepted March $23^{\text {rd }}, 2013$ \\ Copyright (C) 2013 Shin-Ichiro Masunaga et al. This is an open access article distributed under the Creative Commons Attribution \\ License, which permits unrestricted use, distribution, and reproduction in any medium, provided the original work is properly cited.
}

\begin{abstract}
The aim in this study is to examine the effect of tirapazamine (TPZ) and mild temperature hyperthermia (MTH) on the repair of radiation-induced damage in pimonidazole-unlabeled quiescent $(\mathrm{Q})$ tumor cells. Labeling of proliferating $(\mathrm{P})$ cells in C57BL/6J mice bearing EL4 tumors was achieved by continuous administration of 5-bromo-2-deoxyuridine (BrdU). Tumors were irradiated with $\gamma$-rays at $1 \mathrm{~h}$ after the administration of pimonidazole followed by TPZ treatment or MTH. Twenty-four hours later, assessment of the responses of Q and total (= P + Q) cells were based on the frequencies of micronucleation and apoptosis using immunofluorescence staining for BrdU. The response of the pimonidazole-unlabeled tumor cell fractions was assessed by means of apoptosis frequency using immunofluorescence staining for pimonidazole. With $\gamma$-rays only, the pimonidazole-unlabeled cell fraction showed significantly enhanced radio-sensitivity compared with the whole cell fraction more remarkably in Q cells than total cells. However, a significantly greater decrease in radio-sensitivity in the pimonidazole-unlabeled than the whole cell fraction, evaluated using a delayed assay, was more clearly observed in Q cells than total cells. Post-irradiation MTH more remarkably repressed the decrease in radio-sensitivity in the Q cell than the total cells. Post-irradiation TPZ administration produced a large radio-sensitizing effect on both total and Q cells, especially on Q cells. On the other hand, in pimonidazole-unlabeled cell fractions in both total and Q cells, TPZ suppressed the reduction in sensitivity due to delayed assay much more efficiently than MTH, whereas no radio-sensitizing effect was produced. Not only through suppressing the recovery from radiation-induced damage, but also through radio-sensitizing effect, post-irradiation TPZ administration is very useful for repressing the increase in the difference in radio-sensitivity due to the delayed assay, not only between total and Q tumor cells, but also between the pimonidazole-unlabeled and the whole cell fractions within the total and Q tumor cells.
\end{abstract}

Keywords: Quiescent Cell; Recovery from Radiation-Induced Damage; Tirapazamine; Pimonidazole; Mild Temperature Hyperthermia

\section{Introduction}

Human solid tumors are thought to contain moderately large fractions of quiescent $(\mathrm{Q})$ tumor cells, which are not involved in the cell cycle and have stopped dividing, but which are as viable as established experimental animal tumor lines [1]. The presence of $\mathrm{Q}$ cells is probably due, at least in part, to hypoxia and the depletion of nutrition in the tumor core as a consequence of poor

${ }^{*}$ Corresponding author. vascular supply [1]. As a result, with the exception of non-viable $\mathrm{Q}$ cells at the very edge of the necrotic rim where there is diffusion-limited hypoxia, $\mathrm{Q}$ cells are viable and clonogenic but have ceased dividing.

Using our method for selectively detecting the response of Q cells in solid tumors to treatment that damages DNA, the Q cell population in solid tumors has been shown to exhibit more resistance to conventional radioand chemotherapy [2]. The Q cell population has also been demonstrated to have greater capacity to recover 
from radiation- and chemotherapeutic agent-induced damage and to have a significantly larger hypoxic fraction (HF) irrespective of the p53 status of tumor cells [2]. However, the Q tumor cell population has never been shown to be fully hypoxic [2]. Actually, the size of the HF of Q cell populations in SCC VII squamous cell carcinomas, implanted in the hind legs of $\mathrm{C} 3 \mathrm{H} / \mathrm{He}$ mice and with a diameter of $1 \mathrm{~cm}$, was $55.1 \pm 6.2($ mean $\pm \mathrm{SE}$ )\% [3]. Thus, this value was significantly less than $100 \%$, indicating that the $\mathrm{Q}$ cell population undoubtedly includes oxygenated tumor cells.

A few years ago, the universal detection of hypoxic cells in both tissues and cell cultures became possible using pimonidazole, a substituted 2-nitroimidazole, and a mouse IgG1 monoclonal antibody (MAb1) to stable covalent adducts formed through reductive activation of pimonidazole in hypoxic cells [4]. Here, we tried to selectively detect the response of the pimonidazole-unlabeled and probably oxygenated cell fraction of the Q cell population. To achieve this we combined our method for selectively detecting the response of Q cells in solid tumors with the method for detecting cell and tissue hypoxia using pimonidazole and MAb1 to pimonidazole.

The development of bioreductive agents that are particularly toxic to hypoxic cells is considered a promising approach to solving the problem of radio-resistant tumor hypoxia in cancer radiotherapy [5]. Tirapazamine (TPZ), a lead compound in the development of bioreductive hypoxic cytotoxins, in combination with radiation has been shown to be very useful for controlling solid tumors as a whole, especially for controlling $\mathrm{Q}$ tumor cell populations that are rich in hypoxic region [2,5]. Mild temperature hyperthermia (MTH) was reported to increase the response of tumors to radiation by improving oxygennation through an increase in tumour blood flow [6]. Further, MTH was also shown to enhance the tumor response, especially of the intratumor Q cell population, to TPZ [7].

In the present study, the radio-sensitivity of the pimonidazole-unlabeled cell fraction of the Q cell population after cobalt-60 $\gamma$-ray irradiation followed with or without post-irradiation TPZ administration or MTH, was determined, to examine the effect of TPZ and MTH on the repair of radiation-induced damage in pimonidazole-unlabeled Q tumor cell population.

\section{Materials and Methods}

\subsection{Mice and Tumors}

EL4 lymphoma cells (Cell Resource Center for the Biomedical Research Institute of Development, Aging and Cancer, Tohoku University) derived from C57BL/6J mice were maintained in vitro in RPMI 1640 medium supplemented with $12.5 \%$ fetal bovine serum. The p53 status of the EL4 tumor cells was the wild type [8]. Cells were collected from exponentially growing cultures and approximately $1.0 \times 10^{5}$ tumor cells were inoculated subcutaneously into the left hind legs of 9-week-old singeneic female C57BL/6J mice (Japan Animal Co., Ltd., Osaka, Japan). Fourteen days after the inoculation, the tumors, approximately $1 \mathrm{~cm}$ in diameter, were employed for irradiation in this study, and the body weight of the tumor-bearing mice was $22.1 \pm 2.3 \mathrm{~g}$. Mice were handled according to the Recommendations for Handling of Laboratory Animals for Biomedical Research, compiled by the Committee on Safety Handling Regulations for Laboratory Animal Experiments.

\subsection{Labeling with 5-Bromo-2'-Deoxyuridine (BrdU)}

Nine days after the tumor inoculation, mini-osmotic pumps (Durect Corporation, Cupertino, CA) containing BrdU dissolved in physiological saline $(250 \mathrm{mg} / \mathrm{ml})$ were implanted subcutaneously to enable the labeling of all $\mathrm{P}$ cells over a 5-day period. The percentage of labeled cells after continuous labeling with BrdU was $66.1 \% \pm 3.8 \%$ and plateau at this stage. Therefore, tumor cells not incorporating BrdU after continuous exposure were regarded as $Q$ cells.

\subsection{Treatment}

After the labeling with BrdU, tumor-bearing mice received $\gamma$-ray irradiation. $\gamma$-Ray irradiation was performed with a cobalt-60 $\gamma$-ray irradiator at a dose rate of $2.75 \mathrm{~Gy} / \mathrm{min}$ with tumor-bearing mice held in a specially constructed device with the tail firmly fixed with an adhesive tape. In addition, TPZ dissolved in physiological saline was administered at a dose of 224 micromoles $/ \mathrm{kg}(40 \mathrm{mg} / \mathrm{kg})$ singly by intra-peritoneal injection or MTH $\left(40^{\circ} \mathrm{C}\right.$ for $\left.60 \mathrm{~min}\right)$ was also performed immediately after irradiation.

Concerning MTH, the tumors grown in the left hind legs of mice were heated at $40^{\circ} \mathrm{C}$ for $60 \mathrm{~min}$ by immersing the tumor-bearing foot in a water bath. The mouse was held in a specially constructed device with the tail and right leg firmly fixed with an adhesive tape. The left tumor-bearing leg was pulled down by a special sinker (approximately $45 \mathrm{~g}$ ) which was affixed to the skin of the toe with Superglue (Arone-arufa, Konishi Co., Osaka, Japan). The mice were then placed on a circulating water bath maintained at the desired temperature. The mice were air-cooled during the heat treatment [9]. Temperatures at the tumor center equilibrated within 3 to 4 min after immersion in the water bath and remained $0.2^{\circ} \mathrm{C}-0.3^{\circ} \mathrm{C}$ below the bath's temperature. The water 
bath's temperature was maintained at $0.3^{\circ} \mathrm{C}$ above the desired tumor temperature.

Each irradiation group also included mice that were not pretreated with BrdU.

\subsection{Immunofluorescence Staining of BrdU-Labeled and/or Pimonidazole- Labeled Cells and the Observation of Apoptosis and Micronucleation}

Based on our previous report related to the determination of the timing of apoptosis [10], as an immediate assay, an apoptosis assay was undertaken at $6 \mathrm{~h}$ after irradiation and a micronucleus assay was carried out immediately after irradiation. Tumors were excised from mice given BrdU, weighed, minced and trypsinized (0.05\% trypsin and $0.02 \%$ ethylenediamine-tetraacetic acid (EDTA) in phosphate-buffered saline (PBS) at $37^{\circ} \mathrm{C}$ for $20 \mathrm{~min}$ ). Furthermore, as a delayed assay, tumors were also excised from mice given BrdU, weighed, minced and trypsinized at $30 \mathrm{~h}$ after irradiation for the apoptosis assay, and at $24 \mathrm{~h}$ after irradiation for the micronucleus assay. For the apoptosis assay, single cell suspensions were fixed without further treatment. For the micronucleus assay, tumor cell suspensions were incubated for $72 \mathrm{~h}$ in tissue culture dishes containing complete culture medium and $1.0 \mu \mathrm{g} / \mathrm{ml}$ of cytochalasin-B, to inhibit cytokinesis while allowing nuclear division. The cultures were then trypsinized and cell suspensions were fixed. For both assays, after the centrifugation of fixed cell suspensions, the cell pellet was resuspended with cold Carnoy's fixative (ethanol:acetic acid $=3: 1$ in volume). The suspension was placed on a glass microscope slide and the sample was dried at room temperature. Slides were treated with $2 \mathrm{M}$ hydrochloric acid for $60 \mathrm{~min}$ at room temperature to dissociate the histones and partially denature the DNA. They were then immersed in boraxborate buffer ( $\mathrm{pH}$ 8.5) to neutralize the acid. BrdUlabeled tumor cells were detected using indirect immunofluorescence staining with a rat monoclonal anti-BrdU antibody (Abcam plc, Cambridge, UK) and a goat Alexa Fluor 488-conjugated anti-rat IgG antibody (Invitrogen Corp., Carlsbad, CA, USA). Pimonidazole-labeled tumor cells were detected using indirect immunofluorescence staining with a mouse monoclonal antipimonidazole antibody (Hypoxyprobe Inc., Burlington, MA, USA) and a rabbit Alexa Fluor 594-conjugated anti-mouse IgG antibody (Invitrogen Corp., Carlsbad, CA, USA). To enable the observation of the triple staining of tumor cells with green-emitting Alexa Fluor 488 and red-emitting Alexa Fluor 594, cells on the slides were treated with blue-emitting 4'6-diamidino-2-phenylindole (DAPI) (0.5 $\mu \mathrm{g} / \mathrm{ml}$ in PBS) and imaged using a fluorescence microscope.
The frequency of apoptosis in cells not labeled with BrdU ( $=$ Q cells at irradiation) and tumor cells not labeled with pimonidazole was determined by counting apoptotic cells in tumor cells that did not show green fluorescence from Alexa Fluor 488 and red fluorescence from Alexa Fluor 594, respectively. The apoptosis frequency was defined as the ratio of the number of apoptotic cells to the total number of observed tumor cells [9]. The micronucleus frequency in BrdU-unlabeled cells was examined by counting the micronuclei in the binuclear cells that did not show green fluorescence emitted by Alexa Fluor 488. The micronucleus frequency was defined as the ratio of the number of micronuclei in the binuclear cells to the total number of binuclear cells observed [2].

The ratios obtained in tumors not pretreated with BrdU indicated the apoptosis frequency and the micronucleus frequency in the total $(\mathrm{P}+\mathrm{Q})$ tumor cell populations. More than 300 tumor cells and binuclear cells were counted to determine the apoptosis frequency and the micronucleus frequency, respectively.

\subsection{Clonogenic Cell Survival Assay}

The clonogenic cell survival assay was also performed in mice given no BrdU or pimonidazole using an in vivo-in vitro assay method. Tumors were disaggregated by stirring for $20 \mathrm{~min}$ at $37^{\circ} \mathrm{C}$ in PBS containing $0.05 \%$ trypsin and $0.02 \%$ EDTA. The cell yield was $(1.1 \pm 0.3) \times 10^{8} / \mathrm{g}$ tumor weight. A colony formation assay using the in vivo-in vitro assay method was performed with the culture medium mixed with methylcellulose (15.0 g/L) (Aldrich, Milwaukee, WI, USA).

The apoptosis and micronucleus frequencies and surviving fractions for the total cell population were obtained from cells in tumors that were not pretreated with BrdU or pimonidazole. The apoptosis and micronucleus frequencies for $\mathrm{Q}$ cells were obtained from unlabeled tumor cells after continuous BrdU labeling without pimonidazole loading. The apoptosis frequencies for the total tumor cell populations that were not labeled with pimonidazole were obtained from tumor cells that were not labeled with pimonidazole after pimonidazole loading without BrdU pretreatment. The apoptosis frequencies for Q cells that were not labeled with pimonidazole were obtained from tumor cells that were not labeled with BrdU or pimonidazole after both continuous BrdU labeling and pimonidazole loading. Thus, there was no effect of interaction between BrdU and irradiation or between pimonidazole and irradiation on the values for the apoptosis and micronucleus frequencies and surviving fractions. Incidentally, since the rate of pimonidazole-labeled tumor cells could change during culturing with cytochalasin-B over 3 days, following the production of sin- 
gle tumor cell suspensions by excising and mincing the tumors from mice that underwent pimonidazole loading, the micronucleus frequency for the cell fraction that was not labeled with pimonidazole after pimonidazole loading was not determined. As a consequence, the radiosensitivity of the pimonidazole-unlabeled cell fractions was only determined in relation to apoptosis induction. This was the reason for using the EL4 leukemia cell line with its much greater capacity for the induction of apoptosis than other solid tumor-originating cell lines [10].

\subsection{Data Analysis and Statistics}

More than three tumor-bearing mice were used to assess each set of conditions and each experiment was repeated at least twice. To examine the differences between pairs of values, Student's $t$-test was used when variances of the two groups were assumed to be equal with Shapiro-Wilk normality test; otherwise the Welch $t$-test was used.

\section{Results}

Table 1 shows the surviving fractions (SFs) for the total tumor cell population and the $\mathrm{MN}$ and apoptosis frequencies without $\gamma$-ray radiation for the total and Q cell populations. Actually, all the values for the combination with TPZ and MTH were determined 24 hours after TPZ administration and MTH, respectively. Q cells showed significantly higher $\mathrm{MN}$ and apoptosis frequencies than the total cell population under each set of conditions $(P<$ 0.05). Further, although not significantly, pimonidazoleunlabeled cell fraction showed lower apoptosis frequencies than whole tumor cell fraction in both total and Q cell populations. TPZ induced significantly lower SFs and significantly higher $\mathrm{MN}$ and apoptosis frequencies in both the total and $\mathrm{Q}$ cell populations than absolutely no treatment $(P<0.05)$. Again although not significantly, MTH also induced lower SFs and significantly higher $\mathrm{MN}$ and apoptosis frequencies in both the total and Q cell populations than absolutely no treatment.

Cell survival curves for the total tumor cell population as a function of radiation dose are shown in Figure 1. The SFs increased in the following order: $24 \mathrm{~h}$ after irradiation with TPZ < immediately after irradiation alone $<$ $24 \mathrm{~h}$ after irradiation with MTH $<24 \mathrm{~h}$ after irradiation alone.

For baseline correction, we used the net micronucleus frequency to exclude the micronucleus frequency in nonirradiated tumors. The net micronucleus frequency was defined as the micronucleus frequency in the irradiated tumors minus the micronucleus frequency in the nonirradiated tumors. Dose response curves for the net micronucleus frequency in total and Q tumor cell populations as a function of radiation dose are shown in Figure
Table 1. Surviving fraction and micronucleus frequency at 0 Gy.

\begin{tabular}{|c|c|c|}
\hline & Total tumor cells & Quiescent cells \\
\hline \multicolumn{3}{|c|}{$<$ Plating efficiency (\%) > } \\
\hline Without drug or $\mathrm{MTH}^{\mathrm{a}}$ & $25.5 \pm 3.8^{\mathrm{b}}$ & ---- \\
\hline With tirapazamine & $18.4 \pm 2.8$ & --- \\
\hline With MTH & $23.4 \pm 3.8$ & --- \\
\hline \multicolumn{3}{|c|}{$<$ Micronucleus frequency $>$} \\
\hline Without drug or MTH & $0.023 \pm 0.003$ & $0.033 \pm 0.006$ \\
\hline With tirapazamine & $0.049 \pm 0.005$ & $0.134 \pm 0.013$ \\
\hline With MTH & $0.039 \pm 0.004$ & $0.089 \pm 0.008$ \\
\hline \multicolumn{3}{|c|}{$<$ Apoptosis frequency $>$} \\
\hline Without drug or MTH & $0.021 \pm 0.002$ & $0.037 \pm 0.004$ \\
\hline With tirapazamine & $0.027 \pm 0.003$ & $0.044 \pm 0.004$ \\
\hline With MTH & $0.021 \pm 0.002$ & $0.032 \pm 0.003$ \\
\hline \multicolumn{3}{|c|}{ Especially in pimonidazole-unlabeled cell fraction } \\
\hline Without drug or MTH & $0.017 \pm 0.002$ & $0.028 \pm 0.003$ \\
\hline With tirapazamine & $0.024 \pm 0.003$ & $0.035 \pm 0.003$ \\
\hline With MTH & $0.018 \pm 0.002$ & $0.029 \pm 0.003$ \\
\hline
\end{tabular}

${ }^{\mathrm{a}}$ Mild temperature hyperthermia; ${ }^{\mathrm{b}}$ Mean \pm standard error $(\mathrm{n}=9)$. The values for the combination with tirapazamine and MTH were determined 24 hours after tirapazamine administration and MTH, respectively.

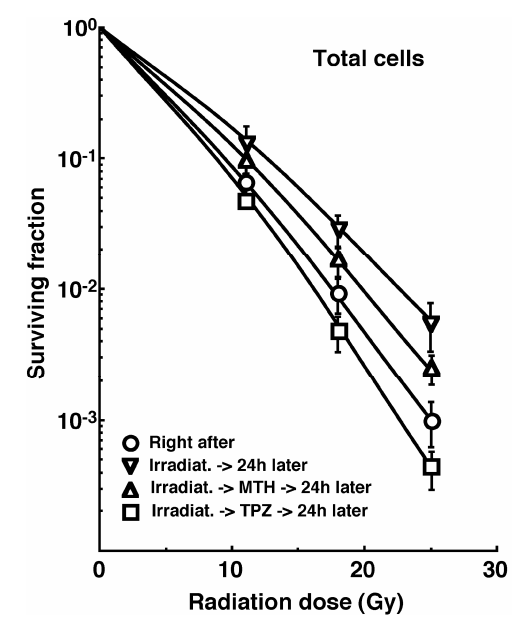

Figure 1. Cell survival curves for the whole tumor cell fraction in the total tumor cell population of EL4 tumors as a function of $\gamma$-ray radiation dose. Circles, reverse triangles, triangles and squares represent the surviving fractions immediately after $\gamma$-ray irradiation only, at $24 \mathrm{~h}$ after $\gamma$-ray irradiation only, at $24 \mathrm{~h}$ after $\gamma$-ray irradiation followed by mild temperature hyperthermia (MTH), and at $24 \mathrm{~h}$ after $\gamma$-ray irradiation followed by tirapazamine (TPZ) administration, respectively. Bars represent standard errors $(n=9)$.

2. Overall, the net micronucleus frequencies were significantly lower in the Q cells than the total cell popula- 
tion $(P<0.05)$. In both the total and $\mathrm{Q}$ cell populations, the net micronucleus frequencies decreased in the following order: $24 \mathrm{~h}$ after irradiation with TPZ $>$ immediately after irradiation alone $>24 \mathrm{~h}$ after irradiation with MTH $>24 \mathrm{~h}$ after irradiation alone.

For another baseline correction, we used the net apoptosis frequency to exclude the apoptosis frequency in non-irradiated tumors. The net apoptosis frequency was the apoptosis frequency in the irradiated tumors minus that in the non-irradiated tumors. Dose response curves for the net apoptosis frequency in the total and Q tumor cell populations as a function of radiation dose are shown in Figure 3. Overall, the net apoptosis frequencies were significantly lower in the $\mathrm{Q}$ than total cell population $(P<$ 0.05). Moreover, the net apoptosis frequency was significantly higher for the cell fraction that was not labeled with pimonidazole than for the whole tumor cell fraction in both the Q and total cell populations under each set of conditions $(P<0.05)$. For the whole cell fractions, in the $\mathrm{Q}$ as well as total tumor cell population, the net apoptosis frequencies decreased in the following order: $24 \mathrm{~h}$ after irradiation with TPZ > immediately after irradiation alone $>24 \mathrm{~h}$ after irradiation with MTH $>24 \mathrm{~h}$ after irradiation alone. However, for the pimonidazole-unlabeled

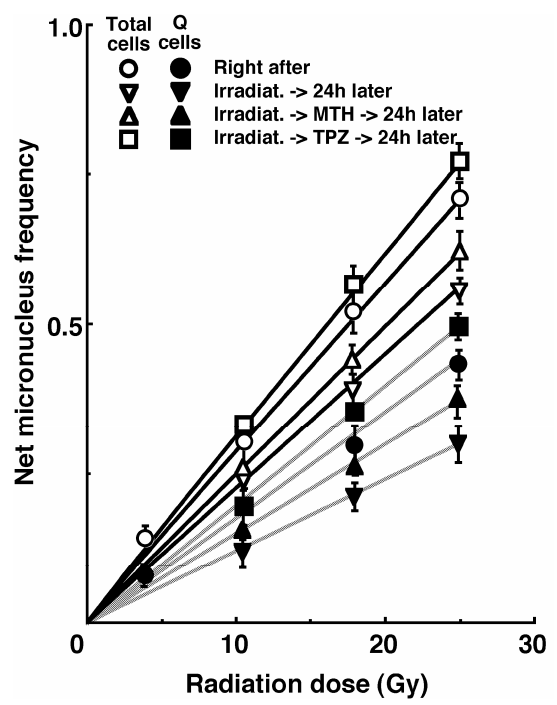

Figure 2. Dose response curves of the net micronucleus frequency for the whole tumor cell fraction in the total and quiescent (Q) tumor cell populations of EL4 tumors as a function of $\gamma$-ray radiation dose. Open and solid symbols represent the net micronucleus frequencies for total and $\mathbf{Q}$ tumor cell populations, respectively. Circles, reverse traingles, triangles and squares represent the surviving fractions immediately after $\gamma$-ray irradiation only, at $24 \mathrm{~h}$ after $\gamma$-ray irradiation only, at $24 \mathrm{~h}$ after $\gamma$-ray irradiation followed by mild temperature hyperthermia (MTH), and at $24 \mathrm{~h}$ after $\gamma$-ray irradiation followed by tirapazamine (TPZ) administration, respectively. Bars represent standard errors $(n=$ 9).

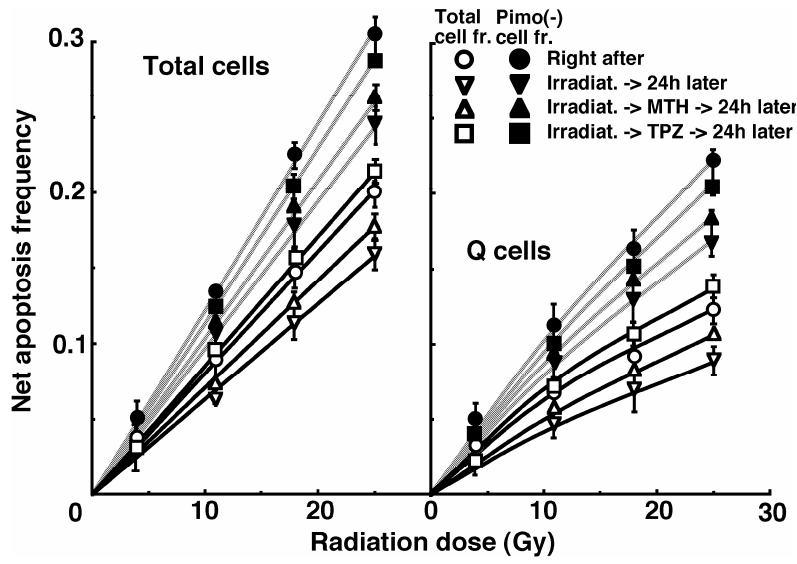

Figure 3. Dose response curves for the net apoptosis frequency of the total (left panel) and quiescent (Q) (right panel) tumor cell populations of EL4 tumors as a function of $\gamma$-ray radiation dose. Open and solid symbols represent the net apoptosis frequencies for the whole tumor cell fraction and the cell fraction not labeled with pimonidazole (Pimo(-)) in both the total and $\mathbf{Q}$ tumor cell populations, respectively. Circles, reverse triangles, triangles and squares represent the surviving fractions immediately after $\gamma$-ray irradiation only, at $24 \mathrm{~h}$ after $\gamma$-ray irradiation only, at $24 \mathrm{~h}$ after $\gamma$-ray irradiation followed by mild temperature hyperthermia (MTH), and at $24 \mathrm{~h}$ after $\gamma$-ray irradiation followed by tirapazamine (TPZ) administration, respectively. Bars represent standard errors $(n=9)$.

cell fractions, in the $\mathrm{Q}$ as well as total tumor cell population, the net apoptosis frequencies decreased in the following order: immediately after irradiation alone $>24 \mathrm{~h}$ after irradiation with TPZ $>24 \mathrm{~h}$ after irradiation with MTH $>24$ h after irradiation alone.

To evaluate the radio-sensitivity of the cell fraction that was not labeled with pimonidazole, as compared with the whole cell fraction in both the total and Q cell populations, dose-modifying factors (DMFs) were calculated using the data shown on Figure 3 (Table 2). Overall, DMF values tended to be higher for the $\mathrm{Q}$ cell than total cell population, and in particular immediately after irradiation with a much larger difference. In both the total and Q cell population, the DMF values had a tendency to decrease in the following order: immediately after irradiation alone $>24 \mathrm{~h}$ after irradiation with MTH $>24 \mathrm{~h}$ after irradiation alone $>24 \mathrm{~h}$ after irradiation with TPZ.

To investigate the reduction in radio-sensitivity caused by a delayed assay, DMFs were calculated using the data given in Figures 1 through 3 (Table 3). On the whole, in the fraction unlabeled with pimonidazole or the whole cell fraction, the values decreased in the following order: after irradiation alone $>24 \mathrm{~h}$ after irradiation with MTH $>24 \mathrm{~h}$ after irradiation with TPZ in both the total and Q cell populations, particularly in the latter population. Under any irradiation condition, all the DMF values for 
Table 2. Dose-mofifying factors for the pimonidazole unlabeled cell fraction compared with the whole cell fraction in the total or quiescent cell population ${ }^{\mathrm{a}}$.

\begin{tabular}{|c|c|c|}
\hline & Total cells & Quiescent cells \\
\hline \multicolumn{3}{|c|}{ Immediately after irradiation } \\
\hline & $1.5 \pm 0.1^{\mathrm{b}}$ & $1.8 \pm 0.15$ \\
\hline \multicolumn{3}{|c|}{24 hours after irradiation } \\
\hline Without drug or $\mathrm{MTH}^{\mathrm{c}}$ & $1.3 \pm 0.1$ & $1.4 \pm 0.1$ \\
\hline With tirapazamine & $1.2 \pm 0.1$ & $1.25 \pm 0.1$ \\
\hline With MTH & $1.45 \pm 0.1$ & $1.6 \pm 0.15$ \\
\hline
\end{tabular}

${ }^{a}$ The ratio of the dose of radiation necessary to obtain the net apoptosis frequency of 0.05 in a whole cell fraction to that needed to obtain the frequency in the pimonidazole unlabeled cell fraction; ${ }^{\mathrm{b}}$ Mean \pm standard error ( $\mathrm{n}=9)$; ${ }^{\mathrm{c}}$ Mild temperature hyperthermia.

Table 3. Dose-modifying factors due to a delayed assay ${ }^{a}$.

\begin{tabular}{cccc}
\hline & Without drug & With tirapazamine & With $\mathrm{MTH}^{\mathrm{b}}$ \\
\hline \multirow{4}{*}{$<$ Surviving fraction $=\mathbf{0 . 0 1}>$} \\
Total cells & $1.3 \pm 0.1^{\mathrm{c}} \quad 0.9 \pm 0.05$ & $1.15 \pm 0.05$ \\
& $<$ Net micronucleus frequency $=\mathbf{0 . 2 5}>$ \\
Total cells & $1.3 \pm 0.1$ & $0.9 \pm 0.05$ & $1.15 \pm 0.05$ \\
Quiescent cells & $1.45 \pm 0.1$ & $0.95 \pm 0.05$ & $1.2 \pm 0.05$ \\
& $<$ Net apoptosis frequency $=\mathbf{0 . 0 5}>$ \\
Total cells & $1.25 \pm 0.1$ & $0.9 \pm 0.05$ & $1.15 \pm 0.05$
\end{tabular}

Especially in pimonidazole-unlabeled cell fraction

$\begin{array}{cccc} & 1.3 \pm 0.1 & 1.05 \pm 0.05 & 1.2 \pm 0.05 \\ \text { Quiescent cells } & 1.35 \pm 0.1 & 0.95 \pm 0.05 & 1.2 \pm 0.05\end{array}$

Especially in pimonidazole-unlabeled cell fraction

$1.65 \pm 0.15 \quad 1.1 \pm 0.05 \quad 1.3 \pm 0.1$

${ }^{\mathrm{a}}$ The ratio of the dose of radiation necessary to obtain each end-point with a delayed assay to that needed to obtain each end-point with an assay immediately after irradiation; ${ }^{b}$ Mild temperature hyperthermia; ${ }^{\mathrm{c}}$ Mean \pm standard error $(n=9)$

irradiation with TPZ were less than 1.0 except for the pimonidazole-unlabeled cell fraction. This means that the combination with TPZ produced not only an inhibiting effect on the recovery from radiation-induced damage but also a radio-sensitization effect except in the pimonidazole-unlabeled cell fraction. The DMF values were higher in the Q cell than total cell population in both the pimonidazole-unlabeled and whole cell fractions. In both the total and Q cell populations, the values were higher for pimonidazole-unlabeled cell fractions than whole cell fractions, particularly in the case of the $\mathrm{Q}$ cells.

To examine the difference in radio-sensitivity between the total and Q cell populations, DMFs that allow us to compare the dose of radiation necessary to obtain each end-point in the two cell populations, were calculated using the data in Figures 2 and 3 (Table 4). All DMF values were significantly higher than $1.0(P<0.05)$. The DMF values increased in the following order: $24 \mathrm{~h}$ after irradiation with TPZ < immediately after irradiation alone $<24 \mathrm{~h}$ after irradiation with MTH $<24 \mathrm{~h}$ after irradiation alone. The values were lower for the sub-population that was not labeled with pimonidazole as compared with the whole cell fraction.

\section{Discussion}

In recent years the concept of cancer stem cells (CSCs), or tumor-initiating cells (tumor clonogens), has attracted a great deal of interest because of the potential clinical significance [11]. In part, these cells are thought to exist in a pathophysiological microenvironment where hypoxia, low $\mathrm{pH}$ and nutrient deprivation occur. Under these microenvironmental conditions, dividing tumor cells have also been thought to become quiescent. Actually, a subset of CSCs or tumor clonogens consists of non-dividing quiescent cells [12].

The fraction of cells that were not labeled with pimonidazole showed significantly higher radio-sensitivity than the whole cell fraction in both the Q and total cell populations, and amongst the Q cells in particular (Table 2). This was probably because the pimonidazole-unlabeled cells were more oxygenated than the whole cell fraction, which comprised oxygenated and hypoxic tumor cells, in both the Q and total tumor cell populations [4]. Additionally, the Q cell population as a whole included a larger hypoxic fraction than the total tumor cell population [2]. As shown in Table 3, the pimonidazole-unlabeled cell fraction had a greater recovery capacity than the whole cell fraction, especially in the case of

Table 4. Dose-modifying factors for quiescent cells relative to total tumor cells .

\begin{tabular}{|c|c|c|c|}
\hline \multirow{2}{*}{$\begin{array}{c}\text { Immediately } \\
\text { after irradiation }\end{array}$} & \multicolumn{3}{|c|}{24 hours after irradiation } \\
\hline & Without drug & With tirapazamine & With MTH $^{\mathrm{b}}$ \\
\hline \multicolumn{4}{|c|}{$<$ Net micronucleus frequency $=0.25>$} \\
\hline $1.7 \pm 0.15^{\mathrm{c}}$ & $1.9 \pm 0.2$ & $1.6 \pm 0.15$ & $1.8 \pm 0.15$ \\
\hline \multicolumn{4}{|c|}{$<$ Net apoptosis frequency $=0.05>$} \\
\hline $1.45 \pm 0.1$ & $1.6 \pm 0.15$ & $1.3 \pm 0.1$ & $1.5 \pm 0.1$ \\
\hline \multicolumn{4}{|c|}{ Especially in pimonidazole-unlabeled cell fraction } \\
\hline $1.2 \pm 0.1$ & $1.4 \pm 0.1$ & $1.25 \pm 0.1$ & $1.3 \pm 0.1$ \\
\hline
\end{tabular}


the Q cells. Therefore, the difference in the radio-sensitivity between the pimonidazole-unlabeled and whole cell fractions more dramatically decreased through the delayed assay in the Q than total cells (Table 2). When combined with MTH, the recovery capacity in both the pimonidazole-unlabeled and the whole cell fractions was efficiently suppressed, especially in Q cell population (Table 3). Thus, the decreases in the DMFs for the pimonidazole-unlabeled relative to the whole cell fractions were repressed with MTH, especially in Q cells (Table 2). In contrast, when combined with TPZ, in the whole cell fractions, the recovery capacity was not only suppressed efficiently but also the radio-sensitizing effect was produced more markedly in Q than total cells. Meanwhile, in the pimonidazole-unlabeled cell fraction, the recovery capacity was suppressed efficiently especially in Q cell population but no radio-sensitizing effect was produced. Consequently, the decreases in the DMFs for the pimonidazole-unlabeled relative to the whole cell fractions were accelerated with TPZ in both total and Q tumor cells, leading to the approach to 1.0 of the DMFs, that is, reaching the approximation in the radio-sensitiveity in both the pimonidazole-unlabeled and the whole cell fractions within the total and Q tumor cells.

On the other hand, the $\mathrm{Q}$ cell population as a whole showed a greater recovery capacity than the total tumor cell population, and the combination with MTH showed a greater potential to suppress a recovery capacity in $\mathrm{Q}$ than total tumor cell population (Table 3). Therefore, the increases in the DMFs for the Q relative to total tumor cell populations were repressed with MTH. When combined with TPZ, in the whole cell fractions, not only the suppressing effect on the recovery capacity but also the radio-sensitizing effect were more markedly observed in Q than total tumor cells, also resulting in repressing the increase in the DMFs due to delayed assay. In the pimonidazole-unlabeled cell fraction, the clearer suppressing effect on the recovery capacity in Q than total tumor cells also lead to repressing the increase in the DMFs due to delayed assay. Furthermore, the repressing effect on the increase in the DMFs due to the delayed assay was more clearly observed through the combination with TPZ than with MTH. Not only through suppressing the recovery from radiation-induced damage but also through radio-sensitizing effect, post-irradiation administration of TPZ is very useful for repressing the increase in the difference in radio-sensitivity due to the delayed assay not only between total and Q tumor cells but also between the pimonidazole-unlabeled and the whole cell fractions within the total and Q tumor cell populations. In other words, the use of TPZ combined with radiation is thought to be very useful for controlling solid tumors through killing tumor cells as homogeneously as possible and overcoming heterogeneous response of malignant tumor cells depending on various intratumor microenvironmental conditions such as oxygenation status [1].

Here, the employed irradiation dose rate was constant (2.75 Gy/min). Incidentally, when $\gamma$-rays alone were delivered without any further treatment at the lower dose rate $(0.039 \mathrm{~Gy} / \mathrm{min})$, the changes in the radio-sensitivity in the pimonidazole-unlabeled and the whole cell fractions within the total and Q cell populations were almost similar to those observed here following $\gamma$-ray irradiation with a high dose rate $(2.75 \mathrm{~Gy} / \mathrm{min})$ [13]. However, the changes in the radio-sensitivity have not yet been determined so far when TPZ or MTH was combined with the low dose rate $\gamma$-ray irradiation. In near future, we would like to analyze these changes in the radio-sensitivity both in the pimonidazole-unlabeled and the whole cell fractions within the total and Q tumor cell populations by all means.

The microenvironmental conditions under which dividing tumor cells become quiescent might promote the formation of micronuclei and apoptosis at $0 \mathrm{~Gy}$ in the whole Q tumor cell fractions, partly due to hypoxic stress (Table 1) [1]. In this study, the Q cells were shown to be significantly less radiosensitive and to have a greater recovery capacity than the total cell population (Figures 2 and 3, Table 4). This finding indicated that more Q cells survive radiation therapy than $\mathrm{P}$ cells. In particular, in the cell fraction that was not labeled with pimonidazole, the difference in radio-sensitivity between the $\mathrm{Q}$ and total cell populations was markedly increased when evaluated using the delayed assay. This was due to the greater recovery capacity of the unlabeled Q cell fraction as compared with the unlabeled cell fraction in the total tumor cell population (Table 3). Therefore, whether in the pimonidazole-unlabeled or the whole Q cell population, it follows that control of the Q cells has a great impact on the outcome of radiation therapy and that the Q cell population can be a critical target in the control of solid tumors.

In this study, the pimonidazole-unlabeled, and probably oxygenated, Q cell fraction showed a greater recovery capacity than the $\mathrm{Q}$ cell population as a whole. However, although there is similarity between the pimonidazole-unlabeled Q cell fraction and CSCs or tumor clonogens in terms of quiescent status and enhanced recovery capacity, CSCs or tumor clonogens are thought to exist under rather hypoxic conditions [11,12,14]. In the future, using human tumor cell lines, the characteristics of the intratumor Q cell population in connection with those of CSCs or tumor clonogens also have to be analyzed.

\section{Acknowledgements}

This study was supported in part by a Grant-in-aid for 
Scientific Research (B) $(23300348,23390355)$ from the Japan Society for the Promotion of Science.

\section{REFERENCES}

[1] P. Vaupel, “Tumor Microenvironmental Physiology and Its Implications for Radiation Oncology,” Seminars in Radiation Oncology, Vol. 14, No. 3, 2004, pp. 198-206. doi:10.1016/j.semradonc.2004.04.008

[2] S. Masunaga and K. Ono, "Significance of the Response of Quiescent Cell Populations within Solid Tumors in Cancer Therapy," Journal of Radiation Research, Vol. 43, No. 1, 2002, pp. 11-25. doi:10.1269/jrr.43.11

[3] S. Masunaga, K. Ono, M. Suzuki, Y. Kinashi and M. Takagaki, "Radiobiologic Significance of Apoptosis and Micronucleation in Quiescent Cells within Solid Tumors Following $\gamma$-Ray Irradiation," International Journal of Radiation Oncology Biology Physics, Vol. 49, No. 5, 2001, pp. 1361-1368. doi:10.1016/S0360-3016(00)01535-2

[4] A. S. Ljungkvis, J. Bussink, P. F. Rijken, J. A. Raleigh, J. Denekamp and A. J. Van der Kogel, "Changes in Tumor Hypoxia Measured with a Double Hypoxic Marker Technique,” International Journal of Radiation Oncology Biology Physics, Vol. 48, No. 5, 2000, pp. 1529-1538. doi:10.1016/S0360-3016(00)00787-2

[5] S. Masunaga, K. Ono and H. Hori, "Exploiting Tumor Hypoxia in the Treatment of Solid Tumors," Japanese Journal of Hyperthermic Oncology, Vol. 17, No. 1, 2001, pp. 13-22.

[6] R. J. Griffin, K. Okajima, A. Ogawa and C. W. Song, "Radiosensitization of Two Murine Tumours with Mild Temperature Hyperthermia and Carbogen Breathing," International Journal of Radiation Biology, Vol. 75, No. 10, 1999, pp. 1299-1306. doi:10.1080/095530099139467

[7] S. Masunaga, K. Ono, H. Hori, Y. Kinashi, M. Suzuki, M. Takagaki, S. Kasai, H. Nagasawa and Y. Uto, "Modification of Tirapazamine-Induced Cytotoxicity in Combination with Mild Hyperthermia and/or Nicotinamide: Reference to Effect on Quiescent Tumour Cells,” International Journal of Hyperthermia, Vol. 15, No. 1, 1999, pp. 7-16. doi:10.1080/026567399285819
[8] S. Masunaga, K. Ono, M. Suzuki, Y. Nishimura, Y. Kinashi, M. Takagaki, H. Hori, H. Nagasawa, Y. Uto, I. Tsuchiya, S. Sadahiro and C. Murayama, "Radiosensitization Effect by Combination with Paclitaxel in Vivo Including the Effect on Intratumor Quiescent Cells,” International Journal of Radiation Oncology Biology Physics, Vol. 50, No. 4, 2001, pp. 1063-1072. doi:10.1016/S0360-3016(01)01553-X

[9] Y. Nishimura, K. Ono, M. Hiraoka, S. Masunaga, S. Jo, Y. Shibamoto, K. Sasai, M. Abe, K. Iga and Y. Ogawa, "Treatment of Murine SCC VII Tumors with Localized Hyperthermia and Temperature-Sensitive Liposomes Containing Cisplatin,” Radiation Research, Vol. 122, No. 2, 1990, pp. 161-167. doi:10.2307/3577601

[10] S. Masunaga, K. Ono, Y. Sakurai, M. Takagaki, T. Kobayashi, Y. Kinashi and M. Suzuki, "Evaluation of Apoptosis and Micronucleation Induced by Reactor Neutron Beams with Two Different Cadmium Ratios in Total and Quiescent Cell Populations within Solid Tumors," International Journal of Radiation Oncology Biology Physics, Vol. 51, No. 3, 2001, pp. 828-839. doi:10.1016/S0360-3016(01)01695-9

[11] C. A. O’Brien, A. Kreso and J. E. Dick, "Cancer Stem Cells in Solid Tumors: An Overview," Seminars in Radiation Oncology, Vol. 19, No. 2, 2009, pp. 71-76. doi:10.1016/j.semradonc.2008.11.001

[12] R. P. Hill, D. T. Marie-Egyptienne and D. W. Hedley, “Cancer Stem Cells, Hypoxia and Metastasis,” Seminars in Radiation Oncology, Vol. 19, No. 2, 2009, pp. 106-111. doi:10.1016/j.semradonc.2008.12.002

[13] S. Masunaga, H. Nagasawa, Y. Liu, Y. Sakurai, H. Tanaka, G. Kashino, M. Suzuki, Y. Kinashi and K. Ono, "Evaluation of the Radiosensitivity of the Oxygenated Tumor Cell Fractions in Quiescent Cell Populations within Solid Tumors," Radiation Research, Vol. 174, No. 4, 2010, pp. 459-466. doi:10.1667/RR2167.1

[14] M. Diehn, R. W. Cho and M. F. Clarke, "Therapeutic Implications of the Cancer Stem Cell Hypothesis," Seminars in Radiation Oncology, Vol. 19, No. 2, 2009, pp. 78-85. doi:10.1016/j.semradonc.2008.11.002 